INPLASY

PROTOCOL

To cite: Liang et al. A Meta

Analysis of the Efficacy of

Traditional Chinese Medicine in

Treating AIDS Related

Diarrhoea. Inplasy protocol

202220120. doi:

10.37766/inplasy2022.2.0120

Received: 28 February 2022

Published: 28 February 2022

Corresponding author:

Biyan Liang

wdlby@126.com

Author Affiliation:

China Academy of Chinese

Medical Sciences.

Support: Science and

Technology Project.

Review Stage at time of this submission: The review has not yet started.

Conflicts of interest:

None declared.

\section{A Meta Analysis of the Efficacy of Traditional Chinese Medicine in Treating AIDS Related Diarrhoea}

\author{
Liang, $\mathrm{B}^{1}$; Zhao, $\mathrm{H}^{2}$; Wang, J33.
}

Review question / Objective: To evaluate the efficacy of traditional Chinese medicine(TCM) in treating AIDS related diarrhoea.

Condition being studied: Chronic diarrhea is one of the most common complications in the occurrence and development of Acquired Immune Deficiency Syndrome(AIDS). It is mainly caused by the destruction of the integrity of the intestinal mucosal barrier of the digestive system and the malabsorption caused by the decline of the immune system function after the patient is infected with human immunodeficiency virus(HIV). The incidence rate in China was $30 \% ? 80 \%$ ?and even $90 \%$ in some backward developing countries. Since 2004, China has implemented the "Four Frees and One Care" policy for HIV/AIDS patients. At present, many scholars had carried out some randomized controlled clinical studies on the efficacy of TCM in treating AIDS related diarrhea, but the quality of these studies was uneven. In order to further explore the clinical efficacy of TCM in treating AIDS related diarrhea, This article searched domestic and foreign randomized controlled trials (RCT) on treating AIDS related diarrhea with TCM, and used meta-analysis methods for evaluation to provide reference for future clinical research.

INPLASY registration number: This protocol was registered with the International Platform of Registered Systematic Review and Meta-Analysis Protocols (INPLASY) on 28 February 2022 and was last updated on 28 February 2022 (registration number INPLASY202220120).

\section{INTRODUCTION}

Review question / Objective: To evaluate the efficacy of traditional Chinese medicine(TCM) in treating AIDS related diarrhoea.
Condition being studied: Chronic diarrhea is one of the most common complications in the occurrence and development of Acquired I m mune Deficiency Syndrome(AIDS). It is mainly caused by the destruction of the integrity of the intestinal 
mucosal barrier of the digestive system and the malabsorption caused by the decline of the immune system function after the patient is infected with human immunodeficiency virus(HIV). The incidence rate in China was 30\%? $80 \%$ ?and even $\mathbf{9 0 \%}$ in some backward developing countries. Since 2004, China has implemented the "Four Frees and One Care" policy for HIV/ AIDS patients. At present, many scholars had carried out some randomized controlled clinical studies on the efficacy of TCM in treating AIDS related diarrhea, but the quality of these studies was uneven. In order to further explore the clinical efficacy of TCM in treating AIDS related diarrhea, This article searched domestic and foreign randomized controlled trials (RCT) on treating AIDS related diarrhea with TCM, and used meta-analysis methods for evaluation to provide reference for future clinical research.

\section{METHODS}

Participant or population: Inclusion criteria:?The research type was RCT of AIDS related diarrhea treated by TCM.?The researchobjects were HIV/AIDS patients and HIV infection-related diseases, regardless of gender and age.? Withdiarrhea symptoms, watery stools at least 3 times a day for more than 2 days (acute diarrhea), or diarrhea atleast 3 times a day for more than 1 month (chronic diarrhea).

Intervention: The intervention measures were the experimental group: TCM treatment. TCM treatments included Chinese patent medicines, herbal medicines, moxibustion, and water acupuncture point injections. Control group: western medicine treatment.

Comparator: The intervention measures were the experimental group: TCM treatment. TCM treatments included Chinesepatent medicines, herbal medicines, moxibustion, and water acupuncture point injections. Control group:western medicine treatment. Internal treatment:Chinese patent medicines, herbal medicines. External treatment: moxibustion, and water acupuncture point injections.

\section{Study designs to be included: RCT.}

Eligibility criteria: (1)The research type was RCT of AIDS related diarrhea treated by TCM.(2)The research objects were HIV/ AIDS patients and HIV infection-related diseases, regardless of gender and age. (3) With diarrhea symptoms, watery stools at least 3 times a day for more than 2 days (acute diarrhea), or diarrhea at least 3 times a day for more than 1 month (chronic diarrhea). (4)The intervention measures were the experimental group: TCM treatment. TCM treatments included Chinese patent medicines, herbal medicines, moxibustion, and water acupuncture point injections. Control group: western medicine treatment.

Information sources: Chinese database

(CBM, CNKI, Wanfang Database) and English database (PubMed, Web of Science, Cochrane Library) were retrieved.

Main outcome(s): The effective rate of related diarrhea.

Quality assessment / Risk of bias analysis: Revman 5.4.1 and Stata17.

Strategy of data synthesis: Revman 5.4.1 and Stata17 were used to meta-analyze the data. Count data utilized relative risk (RR) or odds ratio (OR) and its $95 \%$ confidence interval $(\mathrm{Cl})$ to represent statistical indicators; measurement data used mean difference or standardized mean difference and its $95 \% \mathrm{Cl}$ to represent statistical indicators. Statistical heterogeneity was analyzed by chi-square test. If $P>0.10$ and $I^{2}<50 \%$, it could be considered that multiple similar studies were statistically homogeneous, and a fixed-effect model was used for meta-analysis; if $\mathbf{P}<0.10$ or $I^{2} \geq 50 \%$, there was statistical heterogeneity, and clinically when it was judged that there was clinical consistency between the studies and needed to be merged, a random effects model could be selected; 
descriptive analysis of the data that could not be merged and analyzed. Carry out sensitivity analysis for methodological heterogeneity. Evaluation of bias: Funnel plots and Egger linear regression test were used to evaluate whether there was reporting bias in the analysis results.

Subgroup analysis: Internal treatment, external treatment, internal and external treatment.

Sensitivity analysis: Count data utilized relative risk (RR) or odds ratio (OR) and its 95\% confidence interval $(\mathrm{Cl})$ to represent statistical indicators; measurement data used mean difference or standardized mean difference and its $95 \% \mathrm{Cl}$ to represent statistical indicators.

Country(ies) involved: China.

Keywords: HIV/AIDS, TCM, Diarrhoea, Meta Analysis.

Contributions of each author:

Author 1 - Biyan Liang.

Author 2 - Hanqing Zhao.

Author 3 - Jian Wang. 